\title{
Short-term evaluation of immediately-treated patients with acute HIV infection, recently diagnosed in the National Institute for Infectious Diseases "Prof. Dr. Matei Balş", Bucharest, Romania
}

\author{
Ruxandra Moroti ${ }^{1,2^{*}}$, Adriana Hristea ${ }^{1,2}$, Violeta Molagic ${ }^{2}$, Raluca Jipa ${ }^{2,3}$, Mihaela losipenco ${ }^{2}$, Doina Rîciu ${ }^{2}$, \\ Dan Oțelea², Dragoş Florea ${ }^{1,2}$, Valeriu Gheorghiță2,3, Raluca Hrişcă ${ }^{2,3}$, Ioan Diaconu' ${ }^{2}$, Adrian Streinu-Cercel ${ }^{1,2}$, \\ Otilia Benea ${ }^{1,2}$ \\ From The 10th Edition of the Scientific Days of the National Institute for Infectious Diseases "Prof Dr Matei \\ Bals" \\ Bucharest, Romania. 15-17 October 2014
}

\section{Background}

The rapidly put-on-treatment in acute HIV infection (AHI) seems to achieve functional cure in up to $15 \%$ cases. This represents a huge difference, spontaneous elite-controllers being less than $0.5 \%$.

Objective: To identify the AHI patients and to observe the immune-virological course under immediatelystarted antiretroviral treatment (ART).

\section{Methods}

All newly-diagnosed HIV-infected adults ( $>18$ yo) in the last 18 months (01.2013-06.2014) in an infectious diseases hospital were considered. The including criteria for AHI group were: detectable HIV-RNA or positive antigen/antibody combination assays in the setting of a negative/indeterminate HIV Western blot. AHI group was classified accordingly to Fiebig stages and was further evaluated regarding CD4 count and viral load $(\mathrm{VL})$ at diagnosis, at 3 and 6 months. ART initiation and the regimen were also registered.

\section{Results}

804 adults were newly-diagnosed HIV-positive, out of which 26 patients $(2.32 \%)$ with AHI. The number of

\footnotetext{
* Correspondence: ruxandra_moroti@yahoo.com

${ }^{1}$ Carol Davila University of Medicine and Pharmacy, Bucharest, Romania
} Full list of author information is available at the end of the article patients in Fiebig II/III, IV, V and VI stages was 8, 15, 2 and 1 respectively. The AHI-group had a median age of 31, IQR [25-34] and 3.1:1 male:female ratio. The median CD4 count was 435, IQR [251-775] and the median VL was $5.6 \log 10$, IQR [4.7-7]. Eight out of 26 AHI patients immediately started ART, in Fiebig II/III and IV stages for 7 of them. The $8^{\text {th }}$ received ARV treatment in eclipse phase (for 28 days, as post-exposure prophylaxis) then restarted ART in Fiebig VI stage, at diagnosis moment. The immediately-treated group had a median age of 24 , IQR [20-29], a male:female ratio of 7:1 and all were symptomatic. The median CD4 count at diagnosis was 261, IQR [147-467] and the median VL was $7 \log 10$, IQR [5.6-7], except the partially-treated in eclipse-phase patient, whose CD4 count was 789 and VL was 21977c/ $\mathrm{mL}$ at diagnosis. In the immediately-treated group there was a rise in median CD4 count to 646, IQR [544-764] at month 3 and to 755, IQR [577-950] at month 6 . The median VL declines to $1.6 \log 10$, IQR [1.3-2.2] at month 6 . Five patients received a 3 -drugs regimen and 3 received a 4-drugs regimen. The immune-virological course couldn't be correlated with a particular regimen or with the number of drugs used.

\section{Conclusion}

Two percent of newly diagnosed HIV-infected patient in 18 months in our setting had AHI and one third of 
them received immediate treatment. The short-term benefit was the consistent immune-virological improvement, regardless of ART scheme. However, more than half had detectable VL at month 6, probable due to the very high initial VL.

\section{Authors' details}

${ }^{1}$ Carol Davila University of Medicine and Pharmacy, Bucharest, Romania. ${ }^{2}$ National Institute for Infectious Diseases "Prof. Dr. Matei Balş", Bucharest, Romania. ${ }^{3}$ Central Universitary Emergency Military Hospital Dr Carol Davila, Bucharest, Romania.

Published: 15 October 2014

doi:10.1186/1471-2334-14-S7-O1

Cite this article as: Moroti et al:: Short-term evaluation of immediatelytreated patients with acute HIV infection, recently diagnosed in the National Institute for Infectious Diseases "Prof. Dr. Matei Balş", Bucharest, Romania. BMC Infectious Diseases 2014 14(Suppl 7):O1.

Submit your next manuscript to BioMed Central and take full advantage of:

- Convenient online submission

- Thorough peer review

- No space constraints or color figure charges

- Immediate publication on acceptance

- Inclusion in PubMed, CAS, Scopus and Google Scholar

- Research which is freely available for redistribution

Submit your manuscript at www.biomedcentral.com/submit 